\title{
Review Article \\ Multidisciplinary Treatment for Colorectal Peritoneal Metastases: Review of the Literature
}

\author{
Shaobo Mo ${ }^{1,2}$ and Guoxiang $\mathrm{Cai}^{1,2}$ \\ ${ }^{1}$ Department of Colorectal Surgery, Fudan University Shanghai Cancer Center, Shanghai 200032, China
}

${ }^{2}$ Department of Oncology, Shanghai Medical College, Fudan University, Shanghai 200032, China

Correspondence should be addressed to Guoxiang Cai; gxcai@fudan.edu.cn

Received 16 October 2016; Accepted 22 November 2016

Academic Editor: Amosy M’Koma

Copyright (C) 2016 S. Mo and G. Cai. This is an open access article distributed under the Creative Commons Attribution License, which permits unrestricted use, distribution, and reproduction in any medium, provided the original work is properly cited.

Peritoneum is one of the common sites of metastasis in advanced stage colorectal cancer patients. Colorectal cancer patients with peritoneal metastases (PM) are traditionally believed to have poor prognosis, which indicates it is of no value to adopt surgical treatment. With the advancement of surgical techniques, hyperthermic intraperitoneal chemotherapy (HIPEC), and multidisciplinary treatment in recent years, the cognition and treatment strategies of colorectal peritoneal metastases (CPM) have changed dramatically. In terms of prognosis, CPM under the palliative systemic treatment shows an inferior outcome compared with nonperitoneal metastasis. Nevertheless, some CPM patients amenable to the complete peritoneal cytoreductive surgery (CRS) combined with HIPEC may achieve long-term survival. The prognostic factors of CPM comprise peritoneal carcinomatosis index (PCI), completeness of cytoreduction score (CC score), the presence of extraperitoneal metastasis (liver, etc.), Peritoneal Surface Disease Severity Score (PSDSS), Japanese peritoneal staging, and so forth. Taken together, literature data suggest that a multimodality approach combining complete peritoneal CRS plus HIPEC, systemic chemotherapy, and targeted therapy may be the best treatment option for PM from colorectal cancer.

\section{Introduction}

Peritoneal spread is common in advanced stage colorectal cancer patients and was reported in the past to be associated with a poor prognosis [1-3]. Approximately $5.0 \%$ of colorectal cancer patients present synchronous peritoneal metastases (PM) and about $19.0 \%$ may manifest metachronous disease $[4,5]$. PM is often accompanied by distant metastasis of other organs, such as the liver and lungs. It has been reported that $88 \%$ of colorectal peritoneal metastases (CPM) had other concomitant distant metastases [6]. PM is traditionally perceived as the advanced manifestation of colorectal cancer, with a median survival of 5-7 months, which hardly has the healing possibility and the value of surgical treatment $[1,7,8]$.

With the purpose of seeking more effective treatments for CPM, myriads of approaches have been undertaken over the past decades. Recently, with the accumulation of massive researches on CPM, there is a better understanding of the prognosis of CPM and prognostic influence factors [9]. Moreover, the attitude towards the therapeutic strategies for
CPM has changed tremendously with the update of the concept of multidisciplinary treatment and advances in surgical techniques and hyperthermic intraperitoneal chemotherapy (HIPEC) $[10,11]$. Therefore, the therapeutic efficacy of PM has been greatly improved, achieving better long-term survival in patients with PM from colorectal carcinoma [12].

In this review we commit to exploring the recent advances in multidisciplinary treatment for CPM.

\section{Risks for Development of CPM}

Defining risk factors for the development of CPM is needed to better select patients at high risk for developing PM, which consequently might benefit from intensified adjuvant treatment regimens.

In the population-based study by Segelman et al. [3], independent predictors for developing metachronous CPM were colonic cancer, in particular right-sided, stages T3-T4 tumor, lymph node statuses N1-N2, fewer than 12 lymph 
nodes harvested, emergency procedures, and nonradical resection of the primary tumor ( $\mathrm{R} 1$ or $\mathrm{R} 2)$. From a prospectively expanded single-institutional database with 2406 consecutive patients with colorectal cancer (CRC), clinical and histological data were analyzed for independent risk factors, the results of which demonstrated that age $<62$ years, N2status, T4-status, and location of the primary in the left colon or appendix were independent risk factors for the development of metachronous CPM [13].

The results of study by Lemmens et al. [2] showed that the risk of synchronous CPM was increased in case of advanced T stage, advanced $\mathrm{N}$ stage, poor differentiation grade, younger age, mucinous adenocarcinoma, and right-sided localization of primary tumor. In the study by Jayne and coworkers [1], $53 \%$ of the patients with synchronous PM had a rectal cancer and as for the remaining patients, the primary tumor was located in the left, transverse, and right colon in $25 \%, 7 \%$, and $16 \%$, respectively. According to the study by Sadahiro et al. [14], the primary tumor was most often located in the right colon $(p=0.02)$, with significantly more lymph node metastases in patients with synchronous PM $(p<0.001)$, while the primary tumor was mostly located in the left colon (34\%) in the study of Mulsow et al. [15].

\section{The Prognosis and Prognostic Factors of CPM}

Recent clinical studies show that, receiving palliative systemic treatment alone, patients with CPM have poorer prognosis than those diagnosed with nonperitoneal metastasis. However, the therapeutic approach combining cytoreductive surgery with HIPEC and systemic treatment can achieve long-term survival in appropriate patients with PM from colorectal origin. Furthermore, peritoneal carcinomatosis index (PCI), completeness of cytoreduction score (CC score), liver metastasis, and other related factors are the prognostic influence factors of CPM patients.

3.1. The Prognosis of CPM. A pooled analysis of North Central Cancer Treatment Group Phase III Trials N9741 and N9841 enrolled 2,095 patients with metastatic colorectal cancer (mCRC) and 364 patients with CPM included, receiving palliative chemotherapy (5-fluorouracil, oxaliplatin, or irinotecan). The results of the research showed that patients with CPM shared a significantly shorter median overall survival (OS) (12.7 versus 17.6 months, $p<0.001$ ) and progression-free survival (PFS) (5.8 versus 7.2 months, $p=$ 0.001) compared with nonperitoneal mCRC patients [6]. Similar conclusion can also be drawn in the CAIRO study (previously untreated mCRC patients were treated with chemotherapy) and the CAIRO2 study (previously untreated mCRC patients were treated with chemotherapy and targeted therapy). Klaver et al. [16] reported that no matter how chemotherapy is used alone or combined with targeted therapy, the median OS in patients undergoing CPM were markedly lower than that in patients with mCRC without peritoneal involvement.

CPM patients under the palliative systemic treatment were reported to be connected with poor outcomes, but the prognosis of CPM patients will change noticeably with effective surgical therapy combined with HIPEC. Elias et al. [17] reported the results of their research conducted in French Gustave Roussy Hospital. This research recruited 287 colorectal cancer patients with liver metastases on whom a complete (R0) hepatic lesions resection was performed and 119 cases with PM undergoing peritoneal cytoreductive surgery (CRS) plus HIPEC between 1993 and 2009, excluding patients presenting both liver metastases and PM. The results showed that there were no statistical differences between the liver metastases group and the PM group in 5-year OS rates (38.5\% and 36.5\%, resp.; $p>0.05)$. A study from Australia has also come to the similar conclusion, whose results revealed that the 5-year OS rates of colorectal cancer patients with liver metastases or PM treated with R0 resection of the liver disease or CRS plus HIPEC, respectively, were $33.3 \%$ and $32.1 \%$ ( $p>$ 0.05) [18].

\subsection{The Prognostic Factors of CPM}

3.2.1. Peritoneal Carcinomatosis Index. Peritoneal carcinomatosis index (PCI), a scoring system that quantifies the extent of carcinomatosis, has been recognized as one of the most important prognostic indicators for the long-term outcomes of CPM patients [19-22]. The size and distribution of peritoneal deposits were recorded using the PCI system as described by Glehen et al. [12]. The abdomen is divided into thirteen regions: central region (0), right upper region (1), epigastrium region (2), left upper region (3), left blank region (4), left lower region (5), pelvis region (6), right lower region (7), and right blank region (8), and the small bowel is divided into four: upper jejunum region (9), lower jejunum region (10), upper ileum region (11), and lower ileum region (12) $[12,23]$. Each one is assigned a lesion-size (LS) score of 0 to 3, which would be representative of the largest implant lesion visualized. LS-0 stands for no tumor seen, LS-1 indicates implants $<0.25 \mathrm{~cm}, \mathrm{LS}-2$ indicates implants between 0.25 and $5 \mathrm{~cm}$, and LS-3 indicates implants $>5 \mathrm{~cm}$ or a confluence of disease $[12,23]$. PCI score is a final numerical score of $0-39[12,24]$. One recent study conducted by Faron et al. (2016) demonstrated a perfect linear relationship between the PCI and OS [25]. A survival analysis according to the PCI indicated that the 5-year OS rate was prominently higher in CPM patients with a low $\mathrm{PCI}(<10)$ than in patients with a PCI ranging from 10 to 20 or a PCI of more than 20 (53\%, 23\%, and $12 \%$, resp.; $p<0.001$ ) [26]. Besides, Elias et al. [10] reported a $44 \%$ of 5 -year OS rate with a PCI score of 6 or less and $7 \%$ with a score greater than 19. PCI also influences the likelihood of complete cytoreduction. Most scholars assert that CPM patients with PCI $>20$ have poor oncological outcomes, thus unsuitable for the extensive peritoneal CRS.

3.2.2. Completeness of Cytoreduction Score. Completeness of cytoreduction score (CC score) reflects the thoroughness degree of peritoneal CRS. CC score is calculated according to the maximum diameter of residual tumor after surgery: CC-0 indicates no residual tumor, CC- 1 stands for the maximum diameter of residual tumor $<2.5 \mathrm{~mm}, \mathrm{CC}-2$ stands for the maximum diameter of residual tumor between $2.5 \mathrm{~mm}$ 
and $2.5 \mathrm{~cm}$, and CC-3 stands for the maximum diameter of residual tumor $>2.5 \mathrm{~cm}$ [27]. For colorectal cancer, a complete cytoreduction score includes both CC-0 and CC-1: CC- 0 indicates that all visible tumors are removed completely, and CC-1 presents that only a very small amount of residual tumors that are expected to be eradicated by the HIPEC remain not resected [28]. Complete CRS is not only an important prognostic influence factor but also a necessary requirement for long-term benefit in the management of PM. A French multicentre trial included 523 patients who had undergone operation from 23 centers in four Frenchspeaking countries between 1990 and 2007, the results of which showed remarkably better survival in patients with complete CRS than in those with incomplete CRS [10]. Huang et al. [29] found that CRS plus perioperative intraperitoneal chemotherapy can be safely performed to provide encouraging survival benefits for patients with CPM. Hence, CC- 0 and CC-1 have been recommended to be the standard for CRS by most researches. Conversely, CC- 2 and CC- 3 surgery should not be recommended presently.

3.2.3. Liver Metastasis. It is still controversial when it comes to the prognosis of CPM patients with liver metastasis. On the one hand, the concomitant liver metastasis is a poor prognostic factor. Related researches showed that the prognosis of CPM patients with liver metastasis was conspicuously worse than that of patients with CPM [30]. Elias et al. [10] reported in a multivariate analysis that even if a complete CRS could be obtained with effort, a resectable liver metastasis during the CRS was still a negative prognostic indicator for CPM patients. Glehen et al. [12] confirmed that CPM patients with simultaneous liver metastasis were linked with worse outcomes. On the other hand, a systematic review analyzed the prognosis of colorectal liver metastasis patients with extrahepatic metastasis undergoing surgical resection, the result of which showed that, with the liver resection plus peritoneal CRS for the CPM patients, the 5-year OS rate of $25 \%$ can be materialized [31]. Kianmanesh et al. [32] reported that there were no distinct discrepancies as for the survival rate between patients who underwent CRS plus HIPEC for $\mathrm{PM}$ alone (including the primary resection) and those who had liver metastasis resection (median survival, 35.3 versus 36.0 months, $p=0.73$ ). Some studies have shown that CPM patients with localized PM $(\mathrm{PCI}<12)$ and limited liver metastases (less than 3 metastatic lesions) may benefit from liver metastasis resection and peritoneal CRS [30]. Thus, some authors propose to consider intensive therapy only in patients with $\mathrm{PCI}<12$ and $<3$ liver metastases $[30,33,34]$.

\subsubsection{Other Related Factors. The Peritoneal Surface Disease} Severity Score (PSDSS) was put forward by The American Society of Peritoneal Surface Malignancies [35]. PSDSS consists of 3 prognostic categories: (1) clinical symptoms, (2) extent of peritoneal dissemination (PCI determined on a computed tomographic scan), and (3) primary tumor histology, each of which is subcategorized according to severity [36, 37]. PSDSS can be performed when patients are diagnosed with CPM without the need for intraoperative staging [35]. Clinical analyses testified that PSDSS was an independent prognostic indicator concerning survival not only for patients who underwent a complete CRS and HIPEC, but also for patients treated with systemic chemotherapy without CRS [36, 38-40]. However, although PSDSS is a very interesting system as a recent proposition, it is not used in a common practice and not a rule to be as equivalent as PCI.

The Japanese Society for Cancer of the Colon and Rectum (JSCCR) classifies PM into three subgroups: P1 indicates metastases only to adjacent peritoneum, P2 stands for a few metastases to distant peritoneum, and $\mathrm{P} 3$ represents numerous metastases to distant peritoneum. Relevant researches certified that different $\mathrm{P}$ stage was significantly associated with different prognosis $[5,41]$.

\section{Treatment for CPM}

4.1. Peritoneal CRS. Colorectal peritoneal metastatic lesions and organs involved should be surgically removed during CRS. Apart from peritoneum, greater omentum, lesser omentum, gallbladder, appendix, and ovaries may often be resected, sometimes part of small intestines, rectum and sigmoid colon, uterus, spleen and distal stomach, and so forth as well. Surgeons should attempt to remove all the visible tumors to obtain a complete cytoreduction during peritoneal CRS [34]. Based on current literature, the principle of the CPM resection should meet both the criteria of oncology and the criteria of organ function. The criteria of oncology consist of 3 aspects: (1) PCI $<20$, (2) surgical resection of all the peritoneal metastases or the maximum diameter of residual tumor $<2.5 \mathrm{~mm}$ (CC-0 and CC-1), and (3) R0 resection of the primary tumor and nonperitoneal metastases (liver, lung, or ovary). The criteria of organ function encompass 2 facets: (1) age $<75$ and KPS $>70$ and (2) small intestinal mesentery without severe contracture and no severe small intestinal obstruction. The preoperative assessment of the CPM surgery should include the oncology and the functional evaluation. The oncology evaluations are as follows: (1) tumor antigen (CEA, CA19-9, and CA125), (2) imaging evaluation of peritoneal metastases (enhanced multislice computed tomography (CT) scans plus multiplanar reconstruction, enhanced magnetic resonance imaging (MRI), or positron emission tomography-computed tomography (PET-CT) of abdomen and pelvis), and (3) imaging evaluation of primary tumor and extraperitoneal metastasis. The functional evaluations include gastrointestinal dynamic contrast examination or enhanced CT of the abdomen and pelvis performed to figure out whether there are multisegmental small intestinal obstructions due to small intestinal mesentery contracture.

4.2. HIPEC. HIPEC refers to intraoperative delivery of chemotherapic agents in a recirculating perfusion of the abdominal cavity under hyperthermic conditions [42]. In addition to the traditional superiority of intraperitoneal chemotherapy (abdominal local high concentration, low concentration of peripheral blood, and mild systemic toxicity), HIPEC also has the following advantages: (1) the direct killing effect of hyperthermia, (2) the chemotherapy enhancement effect of hyperthermia, and (3) mechanical flushing effect of irrigation. It can be performed in a closed or open method, 
before or after surgery. The open method makes it more uniform in distribution of heat and chemotherapy drugs, but the operation is relatively inconvenient and heat emission exists. For the closed method, it can reduce the exposure of the medical personnel to chemotherapy; its performance is relatively simple; and the increased abdominal pressure may contribute to the peritoneal permeation of the chemotherapy drugs, whereas the problem is that heat and drugs are not uniformly allocated. At present, there is no gold standard for the chemotherapy drugs used in HIPEC. The American Society of Peritoneal Surface Malignancies (ASPSM) recommends patients to undergo $40 \mathrm{mg}$ mitomycin intraperitoneal perfusion during $90 \mathrm{~min}$ at $42^{\circ} \mathrm{C}$ [43]. Elias et al. [44] from French Gustave Roussy Cancer Campus also reported a series of patients treated with oxaliplatin in the perfusate at $460 \mathrm{mg} / \mathrm{m}^{2}$ in $2 \mathrm{~L} / \mathrm{m}^{2}$ during 30 minutes at $43^{\circ} \mathrm{C}$ and later reported another protocol with oxaliplatin $360 \mathrm{mg} / \mathrm{m}^{2}$ plus irinotecan $360 \mathrm{mg} / \mathrm{m}^{2}$ in $2 \mathrm{~L} / \mathrm{m}^{2}$ of dextrose $5 \%$. During the same time, patients received an intravenous administration of 5-fluorouracil $\left(400 \mathrm{mg} / \mathrm{m}^{2}\right)$ and leucovorin $\left(20 \mathrm{mg} / \mathrm{m}^{2}\right)$.

4.3. Systemic Chemotherapy. CPM patients have a poor prognosis, and the median survival without chemotherapy is about 6 months $[1,7]$. In terms of systemic chemotherapy, there is no difference between CRPC patients and other nonperitoneal metastasis patients, but the effect of systemic chemotherapy is worse in the former compared with that in the latter. In the past few years, new drugs have been introduced into systemic chemotherapy. The application of targeted therapy drugs such as bevacizumab and cetuximab further improves the efficacy of peritoneal metastatic carcinoma. In the retrospective study, receiving chemotherapy in combination with targeted therapy could significantly prolong the OS of CPM patients to 18.2 months, the effect of which is still worse than that of the patients with liver or lung metastases yet [45].

\section{Multidisciplinary Treatment Strategy for CPM}

Complete peritoneal CRS plus HIPEC and systemic treatment (including chemotherapy and targeted therapy) are currently the best modality of multidisciplinary treatment for CPM patients. During CRS, all the visible tumors should be removed to obtain a complete peritoneal CRS. In addition to the cytoreductive surgery, HIPEC is administered to eradicate the tiny and microscopic peritoneal diseases. Systemic chemotherapy is always significant since PM is often part of systemic metastasis. The combined application of these three aspects will probably improve the prognosis of colorectal peritoneal metastatic carcinoma.

According to the National Comprehensive Cancer Network (NCCN) guidelines, combined peritoneal CRS and HIPEC have not been routinely recommended for CPM patients. Nonetheless, the NCCN guidelines also recommend to carry out more well-designed clinical trials. In contrast, the European Society for Medical Oncology (ESMO) guidelines hold more positive attitude towards it. The ESMO guidelines recommend that CPM patients with low PCI should be treated with surgery plus HIPEC when a complete CRS can be obtained. In Japan, a complete CRS has been recommended for patients with P1 phase CPM as well as P2 phase CPM if technically possible [5]. In China, combined peritoneal CRS and HIPEC have also been recommended by experts for CPM patients with PCI $<20$.

Randomized clinical trials have confirmed that combined peritoneal CRS and HIPEC plus systemic chemotherapy is superior to palliative systemic therapy for CPM patients (the median survival, 22.3 and 12.6 months, resp., $p=0.032$ ) [19]. The 5-year survival was $45 \%$ for those patients in whom a complete CRS was achieved [46]. The superiority of combined peritoneal CRS and HIPEC plus systemic chemotherapy can also be clarified in a retrospective study which compared patients undergoing CRS plus HIPEC with comparable controls treated with palliative chemotherapy during the same period but who did not benefit from CRS plus HIPEC because the technique was unavailable in the center at that time. The 5-year OS rates of patients undergoing combined peritoneal CRS and HIPEC plus systemic chemotherapy were significantly higher than that of the patients with palliative systemic therapy (51\% versus $13 \%$, resp.) [47].

For CPM patients with PCI $>20$, peritoneal CRS is not suitable for them currently. However, with the appearance of more effective chemotherapeutic and targeted drugs, it is possible to achieve the reduction of PCI by effective chemotherapy, thereby creating the possibility for further surgery. Because of the high risk of postoperative recurrence of peritoneal metastasis, it is held that CPM patients with PCI $<20$ may benefit from the modality of perioperative chemotherapy.

\section{Controversial Issues and Latest Progress}

6.1. Diffusion-Weighted MRI. Preoperative MRI and CT of the abdomen and pelvis play a critical role in the assessment of the extent of peritoneal and visceral disease in patients being considered for CRS and HIPEC for CPM [48]. In comparison with CT, MRI uses different types of image contrast to create images that are more sensitive for detection of peritoneal lesions [49-51]. With major refinements in hardware and software, functional MRI such as diffusionweighted MRI (DWI) has become technically possible. Various tumors actuate restricted diffusion of water protons which can be evaluated by DWI, resulting in an area of altered signal on DWI [48]. The sensitivity of DWI for depicting peritoneal tumor has opened the gate to potential advancements in detecting disseminated peritoneal metastatic disease. Recently, there have been several very interesting researches regarding DWI [52]. In 2012, Low and Barone [53] calculated the PCI based on DWI before surgery in 35 patients with peritoneal metastatic disease. Compared to surgical-site findings, the overall sensitivity and specificity were $88 \%$ and $74 \%$. Besides, Espada et al. [54] developed a scoring system with a diagnostic accuracy of $91 \%$ by evaluating DWI for detection of PM in 34 patients. In a more recent report [48], preoperative DWI and CT scanning to determine the PCI 
were performed in 22 patients with PM undergoing CRS, where DWI demonstrated predictive accuracy of $91 \%, 83 \%$, and $94 \%$ in overall category, low to moderate tumor burden $(\mathrm{PCI} \leq 20)$, and large tumor burden (PCI $>20)$, compared with CT with a corresponding predictive accuracy of $50 \%$, $50 \%$, and $44 \%$. Of note, it has been reported in 2012 that DWI and 18 fluoro-deoxy-glucose positron emission tomographycomputed tomography (18FDG-PET/CT) showed similar high accuracy in diagnosing peritoneal metastatic carcinoma [55]. Furthermore, DWI appears more sensitive than PET/CT in the supramesocolic area; nevertheless, the limitation of lower sensitivity in the detection of small implants still exists in DWI [55]. Interestingly, a prospective comparison of CT, PET/CT, and whole body DWI in 32 patients conducted by Michielsen et al. [56] in 2014 noted an accuracy for detection of peritoneal disease of $91 \%$ on DWI compared with $75 \%$ on CT and $71 \%$ on FDG-PET/CT, with surgical reference standard in all cases. It is desirable to improve the detection and characterization of peritoneal implants by the simultaneous use of 18FDG-PET and DWI, which remains to be confirmed by future studies $[57,58]$.

6.2. Fluorescence Imaging. Intraoperative fluorescence imaging- (FI-) guided surgery is an emerging technology in the war against cancer that has been proved to improve tumor detection in different tumor types [59-61]. Among the different probes used in identifying neoplasm, indocyanine green (ICG) has been introduced as a safe and useful indicator [61]. Until now, only a few clinical studies have analyzed the potential role of FI taking in PM [62]. Liberale et al. [62] demonstrated in a pilot study that sensitivity and specificity of ICG-FI in patients with nonmucinous CPM were $87.5 \%$ and $100 \%$; contrarily, among the mucinous tumors, the sensitivity of ICG-FI was $0 \%$. Unexpectedly, in 4 out of 14 patients (29\%), additional PM that were not found using visualization and palpation were detected in the surgery modified by intraoperative ICG-FI [62].

6.3. Catumaxomab. Catumaxomab is a trifunctional monoclonal antibody with a mouse-derived anti-EpCAM Fab (fragment antigen-binding) region and a rat anti-CD3 Fab [63]. Catumaxomab antineoplastic activity has been confirmed in vitro, notably in malignant ascites, resulting in a decreased rate of EpCAM plus cells and the release of proinflammatory cytokines (interferon- $\gamma$, tumor necrosis factor$\alpha$, interleukin- (IL-) 2, and IL-6), which enables tumor cells of PM to be specifically identified by catumaxomab via the anti-EpCAM-binding site $[64,65]$. Therefore, intraperitoneal catumaxomab therapy represents a targeted immunotherapy against PM [66]. A randomized phase II/III trial was carried out in 258 patients with symptomatic malignant ascites secondary to EpCAM + carcinomas, to assess the efficacy and safety of intraperitoneal catumaxomab treatment [67]. In this study, patients were randomly assigned to paracentesis plus intraperitoneal catumaxomab or paracentesis alone. The primary endpoint was puncture-free survival used to evaluate the efficacy of intraperitoneal administration of catumaxomab. Puncture-free survival was significantly longer in the group treated with catumaxomab than the control group (46 versus 11 days, $p<0.0001)$. Median overall survival was similar between the two groups (72 versus 68 days, $p=$ 0.0846).

6.4. Systematic Second-Look Surgery and HIPEC. Systematic second-look surgery and HIPEC for CRC patients considered to be at high risk of developing PM have aroused a growing interest among the scientific community. CPM is generally diagnosed in its advanced phase because of the late symptoms onset, low sensitive imaging techniques, and tumor markers. Therefore, the concept of early intervention has emerged. Second-look surgery and HIPEC in patient with a high risk of developing PM treated with curative surgery for CRC have been proposed, but again this would be limited to a small group of patients [68]. In the prospective study, Elias and colleagues reported 29 selected patients at high risk of developing PM without any sign of recurrence on imaging studies who underwent second-look surgery 13 months after resection of the primary tumor. In $55 \%$ of patients, persistent adenocarcinoma was documented. In patients with documented disease at second look 9 of 16 patients were disease-free at 27 months of median follow-up [69]. Besides, patient with or without macroscopic PM at second-look surgery treated with CRS and HIPEC developed a low rate of peritoneal recurrences (17\% at a median follow-up of 30 months) [70]. One ongoing prospective randomized clinical trial is designed in France. All patients at risk will receive the gold standard adjuvant systemic chemotherapy with FOLFOX 6 over 6 months. Patients with a negative followup will be randomly assigned to surveillance or second-look laparotomy and HIPEC. The object of the clinical trial is to evaluate the peritoneal recurrence rate for 3 years.

6.5. The Necessity of HIPEC. CRS plus HIPEC is increasingly being used for the treatment of PM of colorectal origin. However, it is still controversial whether HIPEC is the indispensable cornerstone of the best modality of multidisciplinary treatment for CPM [71]. It is not yet possible to evaluate the morbidity and mortality rates related to HIPEC alone independently of CRS, as both procedures are performed jointly during the same surgery. Only those patients who undergo $\mathrm{CRS}$ can receive HIPEC. The published mortality rates in CRS + HIPEC range from 0 to $11 \%$ (mean 4-6\%) [71]. Survival rates are reported better in patients who undergo CRS + HIPEC versus those who receive systemic chemotherapy [72]. Seeking to elucidate the true role of HIPEC in the context of radical combined therapy, a multicentre, randomized, openlabel study currently in phase III (PRODIGE 7) was designed with parallel groups and two treatment arms comparing CRS + HIPEC + systemic chemotherapy before or after surgery versus CRS + systemic chemotherapy before or after surgery. The final collection date for primary outcome measure was completed in December 2015. Once the results of this study are achieved, we should have considerably more evidence regarding the efficacy of this treatment modality [71].

\section{Conclusion}

Complete peritoneal CRS plus HIPEC and systemic treatment (including chemotherapy and targeted therapy) might 
be the best modality of multidisciplinary treatment for CPM. Patients appropriate for aggressive therapy undergoing complete peritoneal CRS plus HIPEC and systemic treatment can get better long-term outcome. PCI and CC score are important prognostic indicators of CPM patients. More welldesigned clinical trials are in terrible need to figure out the best multidisciplinary treatment modality for CPM.

\section{Competing Interests}

The authors declare that there is no conflict of interests regarding the publication of this paper.

\section{Acknowledgments}

This work was supported by Department of Colorectal Surgery, Fudan University Shanghai Cancer Center.

\section{References}

[1] D. G. Jayne, S. Fook, C. Loi, and F. Seow-Choen, "Peritoneal carcinomatosis from colorectal cancer," British Journal of Surgery, vol. 89, no. 12, pp. 1545-1550, 2002.

[2] V. E. Lemmens, Y. L. Klaver, V. J. Verwaal, H. J. Rutten, J. W. W. Coebergh, and I. H. de Hingh, "Predictors and survival of synchronous peritoneal carcinomatosis of colorectal origin: a population-based study," International Journal of Cancer, vol. 128, no. 11, pp. 2717-2725, 2011.

[3] J. Segelman, F. Granath, T. Holm, M. MacHado, H. Mahteme, and A. Martling, "Incidence, prevalence and risk factors for peritoneal carcinomatosis from colorectal cancer," British Journal of Surgery, vol. 99, no. 5, pp. 699-705, 2012.

[4] Y. R. B. M. van Gestel, I. H. J. T. de Hingh, M. P. P. van HerkSukel et al., "Patterns of metachronous metastases after curative treatment of colorectal cancer," Cancer Epidemiology, vol. 38, no. 4, pp. 448-454, 2014.

[5] T. Watanabe, M. Itabashi, Y. Shimada et al., "Japanese Society for Cancer of the Colon and Rectum (JSCCR) guidelines 2010 for the treatment of colorectal cancer," International Journal of Clinical Oncology, vol. 17, no. 1, pp. 1-29, 2012.

[6] J. Franko, Q. Shi, C. D. Goldman et al., "Treatment of colorectal peritoneal carcinomatosis with systemic chemotherapy: a pooled analysis of north central cancer treatment group phase III trials N9741 and N9841," Journal of Clinical Oncology, vol. 30, no. 3, pp. 263-267, 2012.

[7] B. Sadeghi, C. Arvieux, O. Glehen et al., "Peritoneal carcinomatosis from non-gynecologic malignancies: results of the EVOCAPE 1 multicentric prospective study," Cancer, vol. 88, no. 2, pp. 358-363, 2000.

[8] D. Z. J. Chu, N. P. Lang, C. Thompson, P. K. Osteen, and K. C. Westbrook, "Peritoneal carcinomatosis in nongynecologic malignancy. A prospective study of prognostic factors," Cancer, vol. 63, no. 2, pp. 364-367, 1989.

[9] I. Thomassen, Y. R. Van Gestel, V. E. Lemmens, and I. H. De Hingh, "Incidence, prognosis, and treatment options for patients with synchronous peritoneal carcinomatosis and liver metastases from colorectal origin," Diseases of the Colon and Rectum, vol. 56, no. 12, pp. 1373-1380, 2013.

[10] D. Elias, F. Gilly, F. Boutitie et al., "Peritoneal colorectal carcinomatosis treated with surgery and perioperative intraperitoneal chemotherapy: Retrospective Analysis of 523 Patients From a
Multicentric French Study," Journal of Clinical Oncology, vol. 28, no. 1, pp. 63-68, 2010.

[11] A. Bhatt and D. Goéré, "Cytoreductive surgery plus HIPEC for peritoneal metastases from colorectal cancer," Indian Journal of Surgical Oncology, vol. 7, no. 2, pp. 177-187, 2016.

[12] O. Glehen, F. Kwiatkowski, P. H. Sugarbaker et al., "Cytoreductive surgery combined with perioperative intraperitoneal chemotherapy for the management of peritoneal carcinomatosis from colorectal cancer: a multi-institutional study," Journal of Clinical Oncology, vol. 22, no. 16, pp. 3284-3292, 2004.

[13] A. G. Kerscher, T. C. Chua, M. Gasser et al., "Impact of peritoneal carcinomatosis in the disease history of colorectal cancer management: a longitudinal experience of 2406 patients over two decades," British Journal of Cancer, vol. 108, no. 7, pp. 1432-1439, 2013.

[14] S. Sadahiro, T. Suzuki, Y. Maeda et al., "Prognostic factors in patients with synchronous peritoneal carcinomatosis (PC) caused by a primary cancer of the colon," Journal of Gastrointestinal Surgery, vol. 13, no. 9, pp. 1593-1598, 2009.

[15] J. Mulsow, S. Merkel, A. Agaimy, and W. Hohenberger, "Outcomes following surgery for colorectal cancer with synchronous peritoneal metastases," British Journal of Surgery, vol. 98, no. 12, pp. 1785-1791, 2011.

[16] Y. L. B. Klaver, L. H. J. Simkens, V. E. P. P. Lemmens et al., "Outcomes of colorectal cancer patients with peritoneal carcinomatosis treated with chemotherapy with and without targeted therapy," European Journal of Surgical Oncology, vol. 38, no. 7, pp. 617-623, 2012.

[17] D. Elias, M. Faron, B. S. Iuga et al., "Prognostic similarities and differences in optimally resected liver metastases and peritoneal metastases from colorectal cancers," Annals of Surgery, vol. 261, no. 1, pp. 157-163, 2015.

[18] C. Q. Cao, T. D. Yan, W. Liauw, and D. L. Morris, “Comparison of optimally resected hepatectomy and peritonectomy patients with colorectal cancer metastasis," Journal of Surgical Oncology, vol. 100, no. 7, pp. 529-533, 2009.

[19] V. J. Verwaal, S. van Ruth, E. de Bree et al., "Randomized trial of cytoreduction and hyperthermic intraperitoneal chemotherapy versus systemic chemotherapy and palliative surgery in patients with peritoneal carcinomatosis of colorectal cancer," Journal of Clinical Oncology, vol. 21, no. 20, pp. 3737-3743, 2003.

[20] R. G. da Silva and P. H. Sugarbaker, "Analysis of prognostic factors in seventy patients having a complete cytoreduction plus perioperative intraperitoneal chemotherapy for carcinomatosis from colorectal cancer," Journal of the American College of Surgeons, vol. 203, no. 6, pp. 878-886, 2006.

[21] D. Elias, F. Blot, A. El Otmany et al., "Curative treatment of peritoneal carcinomatosis arising from colorectal cancer by complete resection and intraperitoneal chemotherapy," Cancer, vol. 92, no. 1, pp. 71-76, 2001.

[22] T. D. Yan, F. Chu, M. Links, P. C. Kam, D. Glenn, and D. L. Morris, "Cytoreductive surgery and perioperative intraperitoneal chemotherapy for peritoneal carcinomatosis from colorectal carcinoma: non-mucinous tumour associated with an improved survival," European Journal of Surgical Oncology, vol. 32, no. 10, pp. 1119-1124, 2006.

[23] P. H. Sugarbaker, "Peritoneal surface oncology: review of a personal experience with colorectal and appendiceal malignancy," Techniques in Coloproctology, vol. 9, no. 2, pp. 95-103, 2005.

[24] J.-L. Koh, T. D. Yan, D. Glenn, and D. L. Morris, "Evaluation of preoperative computed tomography in estimating peritoneal 
cancer index in colorectal peritoneal carcinomatosis," Annals of Surgical Oncology, vol. 16, no. 2, pp. 327-333, 2009.

[25] M. Faron, R. Macovei, D. Goéré, C. Honoré, L. Benhaim, and D. Elias, "Linear relationship of peritoneal cancer index and survival in patients with peritoneal metastases from colorectal cancer," Annals of Surgical Oncology, vol. 23, no. 1, pp. 114-119, 2016.

[26] D. Goéré, D. Malka, D. Tzanis et al., "Is there a possibility of a cure in patients with colorectal peritoneal carcinomatosis amenable to complete cytoreductive surgery and intraperitoneal chemotherapy?" Annals of Surgery, vol. 257, no. 6, pp. 1065-1071, 2013.

[27] A. A. K. Tentes, "The management of peritoneal surface malignancy of colorectal cancer origin," Techniques in Coloproctology, vol. 8, no. 1, pp. S39-S42, 2004.

[28] P. H. Sugarbaker and D. P. Ryan, "Cytoreductive surgery plus hyperthermic perioperative chemotherapy to treat peritoneal metastases from colorectal cancer: standard of care or an experimental approach?” The Lancet Oncology, vol. 13, no. 8, pp. e362-e369, 2012.

[29] Y. Huang, N. A. Alzahrani, T. C. Chua, W. Liauw, and D. L. Morris, "Impacts of low peritoneal cancer index on the survival outcomes of patient with peritoneal carcinomatosis of colorectal origin," International Journal of Surgery, vol. 23, pp. 181-185, 2015.

[30] L. Maggiori, D. Goéré, B. Viana et al., "Should patients with peritoneal carcinomatosis of colorectal origin with synchronous liver metastases be treated with a curative intent? A Casecontrol Study," Annals of Surgery, vol. 258, no. 1, pp. 116-121, 2013.

[31] M. Hwang, T. T. Jayakrishnan, D. E. Green et al., "Systematic review of outcomes of patients undergoing resection for colorectal liver metastases in the setting of extra hepatic disease," European Journal of Cancer, vol. 50, no. 10, pp. 1747-1757, 2014.

[32] R. Kianmanesh, S. Scaringi, J.-M. Sabate et al., "Iterative cytoreductive surgery associated with hyperthermic intraperitoneal chemotherapy for treatment of peritoneal carcinomatosis of colorectal origin with or without liver metastases," Annals of Surgery, vol. 245, no. 4, pp. 597-603, 2007.

[33] D. Elias, E. Benizri, M. Pocard, M. Ducreux, V. Boige, and P. Lasser, "Treatment of synchronous peritoneal carcinomatosis and liver metastases from colorectal cancer," European Journal of Surgical Oncology, vol. 32, no. 6, pp. 632-636, 2006.

[34] C. Vallicelli, D. Cavaliere, F. Catena et al., "Management of peritoneal carcinomatosis from colorectal cancer: review of the literature," International Journal of Colorectal Disease, vol. 29, no. 8, pp. 895-898, 2014.

[35] J. Esquivel, A. M. Lowy, M. Markman et al., "The American Society of Peritoneal Surface Malignancies (ASPSM) Multiinstitution Evaluation of the Peritoneal Surface Disease Severity Score (PSDSS) in 1,013 Patients with Colorectal Cancer with Peritoneal Carcinomatosis," Annals of Surgical Oncology, vol. 21, no. 13, pp. 4195-4201, 2014.

[36] J. O. W. Pelz, A. Stojadinovic, A. Nissan, W. Hohenberger, and J. Esquivel, "Evaluation of a peritoneal surface disease severity score in patients with colon cancer with peritoneal carcinomatosis," Journal of Surgical Oncology, vol. 99, no. 1, pp. 9-15, 2009.

[37] J. L. Ng, W. S. Ong, C. S. Chia, G. H. Tan, K. Soo, and M. C. Teo, "Prognostic relevance of the Peritoneal Surface Disease Severity Score compared to the peritoneal cancer index for colorectal peritoneal carcinomatosis," International Journal of Surgical Oncology, vol. 2016, Article ID 2495131, 7 pages, 2016.

[38] T. C. Chua, D. L. Morris, and J. Esquivel, "Impact of the peritoneal surface disease severity score on survival in patients with colorectal cancer peritoneal carcinomatosis undergoing complete cytoreduction and hyperthermic intraperitoneal chemotherapy," Annals of Surgical Oncology, vol. 17, no. 5, pp. 1330-1336, 2010.

[39] J. O. W. Pelz, T. C. Chua, J. Esquivel et al., "Evaluation of best supportive care and systemic chemotherapy as treatment stratified according to the retrospective peritoneal surface disease severity score (PSDSS) for peritoneal carcinomatosis of colorectal origin," BMC Cancer, vol. 10, article 689, 2010.

[40] J. Esquivel, T. C. Chua, A. Stojadinovic et al., "Accuracy and clinical relevance of computed tomography scan interpretation of peritoneal cancer index in colorectal cancer peritoneal carcinomatosis: A Multi-institutional Study," Journal of Surgical Oncology, vol. 102, no. 6, pp. 565-570, 2010.

[41] H. Kobayashi, K. Kotake, K. Funahashi et al., "Clinical benefit of surgery for stage IV colorectal cancer with synchronous peritoneal metastasis," Journal of Gastroenterology, vol. 49, no. 4, pp. 646-654, 2014.

[42] J. S. Spratt, R. A. Adcock, M. Muskovin, W. Sherrill, and J. McKeown, "Clinical delivery system for intraperitoneal hyperthermic chemotherapy," Cancer Research, vol. 40, no. 2, pp. 256260, 1980.

[43] K. Turaga, E. Levine, R. Barone et al., "Consensus guidelines from the American Society of Peritoneal Surface Malignancies on standardizing the delivery of hyperthermic intraperitoneal chemotherapy (HIPEC) in colorectal cancer patients in the United States," Annals of Surgical Oncology, vol. 21, no. 5, pp. 1501-1505, 2014.

[44] D. Elias, L. Sideris, M. Pocard et al., "Efficacy of intraperitoneal chemohyperthermia with oxaliplatin in colorectal peritoneal carcinomatosis. Preliminary results in 24 patients," Annals of Oncology, vol. 15, no. 5, pp. 781-785, 2004.

[45] Y. L. B. Klaver, B. J. M. Leenders, G.-J. Creemers et al., "Addition of biological therapies to palliative chemotherapy prolongs survival in patients with peritoneal carcinomatosis of colorectal origin," American Journal of Clinical Oncology, vol. 36, no. 2, pp. 157-161, 2013.

[46] V. J. Verwaal, S. Bruin, H. Boot, G. Van Slooten, and H. Van Tinteren, "8-Year follow-up of randomized trial: cytoreduction and hyperthermic intraperitoneal chemotherapy versus systemic chemotherapy in patients with peritoneal carcinomatosis of colorectal cancer," Annals of Surgical Oncology, vol. 15, no. 9, pp. 2426-2432, 2008.

[47] D. Elias, J. H. Lefevre, J. Chevalier et al., "Complete cytoreductive surgery plus intraperitoneal chemohyperthermia with oxaliplatin for peritoneal carcinomatosis of colorectal origin," Journal of Clinical Oncology, vol. 27, no. 5, pp. 681-685, 2009.

[48] R. N. Low, R. M. Barone, and J. Lucero, "Comparison of MRI and CT for predicting the Peritoneal Cancer Index (PCI) preoperatively in patients being considered for cytoreductive surgical procedures," Annals of Surgical Oncology, vol. 22, no. 5, pp. 1708-1715, 2015.

[49] R. N. Low, R. M. Barone, J. M. Gurney, and W. D. Muller, "Mucinous appendiceal neoplasms: preoperative MR staging and classification compared with surgical and histopathologic findings," American Journal of Roentgenology, vol. 190, no. 3, pp. 656-665, 2008. 
[50] R. N. Low, C. P. Sebrechts, R. M. Barone, and W. Muller, "Diffusion-weighted MRI of peritoneal tumors: comparison with conventional MRI and surgical and histopathologic findings-a feasibility study," American Journal of Roentgenology, vol. 193, no. 2, pp. 461-470, 2009.

[51] S. Kyriazi, D. J. Collins, V. A. Morgan, S. L. Giles, and N. M. deSouza, "Diffusion-weighted imaging of peritoneal disease for noninvasive staging of advanced ovarian cancer," Radiographics, vol. 30, no. 5, pp. 1269-1285, 2010.

[52] A. G. Rockall, "Diffusion weighted MRI in ovarian cancer," Current Opinion in Oncology, vol. 26, no. 5, pp. 529-535, 2014.

[53] R. N. Low and R. M. Barone, "Combined diffusion-weighted and gadolinium-enhanced MRI can accurately predict the peritoneal cancer index preoperatively in patients being considered for cytoreductive surgical procedures," Annals of Surgical Oncology, vol. 19, no. 5, pp. 1394-1401, 2012.

[54] M. Espada, J. R. Garcia-Flores, M. Jimenez et al., "Diffusionweighted magnetic resonance imaging evaluation of intraabdominal sites of implants to predict likelihood of suboptimal cytoreductive surgery in patients with ovarian carcinoma," European Radiology, vol. 23, no. 9, pp. 2636-2642, 2013.

[55] M. Soussan, G. Des Guetz, V. Barrau et al., "Comparison of FDG-PET/CT and MR with diffusion-weighted imaging for assessing peritoneal carcinomatosis from gastrointestinal malignancy," European Radiology, vol. 22, no. 7, pp. 1479-1487, 2012.

[56] K. Michielsen, I. Vergote, K. Op De Beeck et al., "Whole-body MRI with diffusion-weighted sequence for staging of patients with suspected ovarian cancer: a clinical feasibility study in comparison to CT and FDG-PET/CT," European Radiology, vol. 24, no. 4, pp. 889-901, 2014.

[57] M. Barral, C. Eveno, C. Hoeffel et al., "Diffusion-weighted magnetic resonance imaging in colorectal cancer," Journal of Visceral Surgery, vol. 153, no. 5, pp. 361-369, 2016.

[58] N. F. Schwenzer, H. Schmidt, S. Gatidis et al., "Measurement of apparent diffusion coefficient with simultaneous MR/positron emission tomography in patients with peritoneal carcinomatosis: comparison with 18F-FDG-PET," Journal of Magnetic Resonance Imaging, vol. 40, no. 5, pp. 1121-1128, 2014.

[59] K. Polom, D. Murawa, Y. S. Rho, P. Nowaczyk, M. Hünerbein, and P. Murawa, "Current trends and emerging future of indocyanine green usage in surgery and oncology: a literature review," Cancer, vol. 117, no. 21, pp. 4812-4822, 2011.

[60] B. E. Schaafsma, J. S. D. Mieog, M. Hutteman et al., "The clinical use of indocyanine green as a near-infrared fluorescent contrast agent for image-guided oncologic surgery," Journal of Surgical Oncology, vol. 104, no. 3, pp. 323-332, 2011.

[61] J. A. Zelken and A. P. Tufaro, "Current trends and emerging future of indocyanine green usage in surgery and oncology: an update," Annals of Surgical Oncology, vol. 22, no. 3, pp. 12711283, 2015.

[62] G. Liberale, S. Vankerckhove, M. G. Caldon et al., "Fluorescence imaging after indocyanine green injection for detection of peritoneal metastases in patients undergoing cytoreductive surgery for peritoneal carcinomatosis from colorectal cancer: a pilot study," Annals of Surgery, vol. 264, no. 6, pp. 1110-1115, 2016.

[63] P. Ruf and H. Lindhofer, "Induction of a long-lasting antitumor immunity by a trifunctional bispecific antibody," Blood, vol. 98, no. 8, pp. 2526-2534, 2001.

[64] M. Jäger, A. Schoberth, P. Ruf et al., "Immunomonitoring results of a phase II/III study of malignant ascites patients treated with the trifunctional antibody catumaxomab (Anti-EpCAM x antiCD3)," Cancer Research, vol. 72, no. 1, pp. 24-32, 2012.

[65] M. A. Ströhlein and M. M. Heiss, "Intraperitoneal immunotherapy to prevent peritoneal carcinomatosis in patients with advanced gastrointestinal malignancies," Journal of Surgical Oncology, vol. 100, no. 4, pp. 329-330, 2009.

[66] M. A. Ströhlein and M. M. Heiss, "Immunotherapy of peritoneal carcinomatosis," Cancer treatment and research, vol. 134, pp. 483-491, 2007.

[67] M. M. Heiss, P. Murawa, P. Koralewski et al., "The trifunctional antibody catumaxomab for the treatment of malignant ascites due to epithelial cancer: results of a prospective randomized phase II/III trial," International Journal of Cancer, vol. 127, no. 9, pp. 2209-2221, 2010.

[68] P. H. Sugarbaker, "Second-look surgery for colorectal cancer: revised selection factors and new treatment options for greater success," International Journal of Surgical Oncology, vol. 2011, Article ID 915078, 8 pages, 2011.

[69] D. Elias, D. Goéré, D. Di Pietrantonio et al., "Results of systematic second-look surgery in patients at high risk of developing colorectal peritoneal carcinomatosis," Annals of Surgery, vol. 247, no. 3, pp. 445-450, 2008.

[70] D. Elias, C. Honoré, F. Dumont et al., "Results of systematic second-look surgery plus hipec in asymptomatic patients presenting a high risk of developing colorectal peritoneal carcinomatosis," Annals of Surgery, vol. 254, no. 2, pp. 289-293, 2011.

[71] F. Losa, P. Barrios, R. Salazar et al., "Cytoreductive surgery and intraperitoneal chemotherapy for treatment of peritoneal carcinomatosis from colorectal origin," Clinical and Translational Oncology, vol. 16, no. 2, pp. 128-140, 2014.

[72] J. Franko, Z. Ibrahim, N. J. Gusani, M. P. Holtzman, D. L. Bartlett, and H. J. Zeh III, "Cytoreductive surgery and hyperthermic intraperitoneal chemoperfusion versus systemic chemotherapy alone for colorectal peritoneal carcinomatosis," Cancer, vol. 116, no. 16, pp. 3756-3762, 2010. 


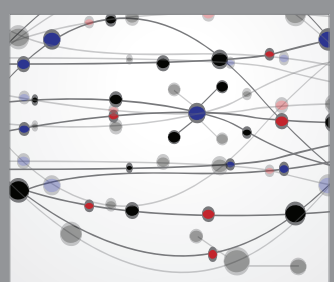

The Scientific World Journal
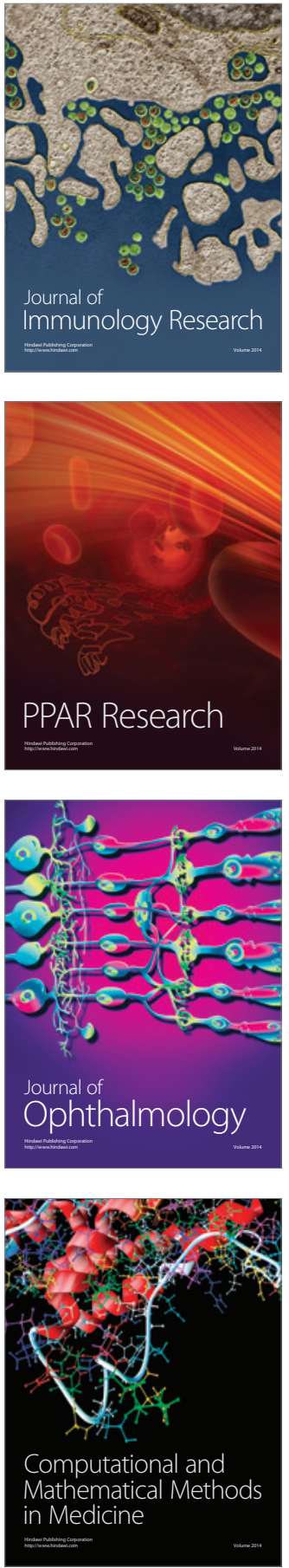

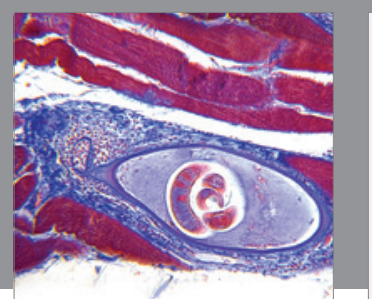

Gastroenterology Research and Practice

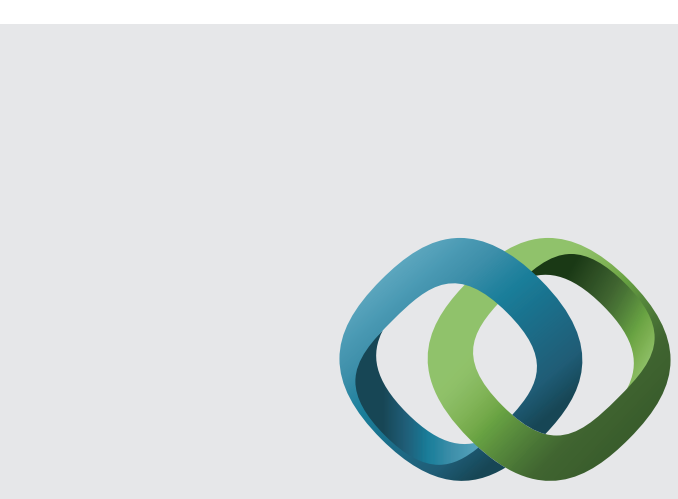

\section{Hindawi}

Submit your manuscripts at

http://www.hindawi.com
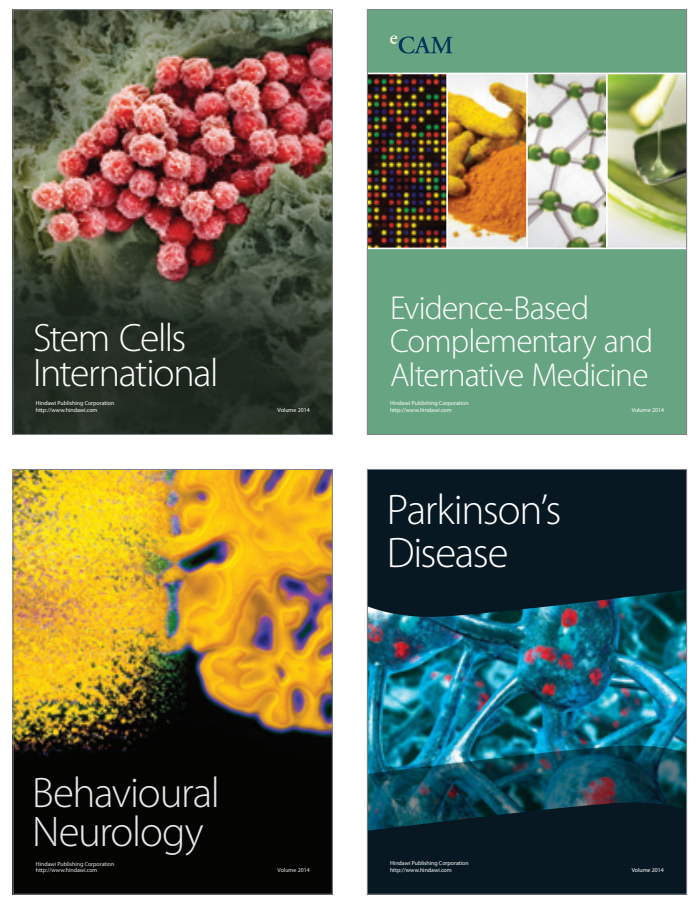
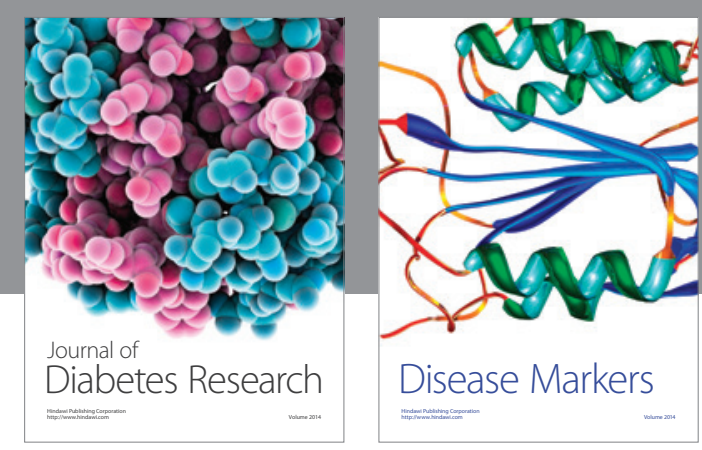

Disease Markers
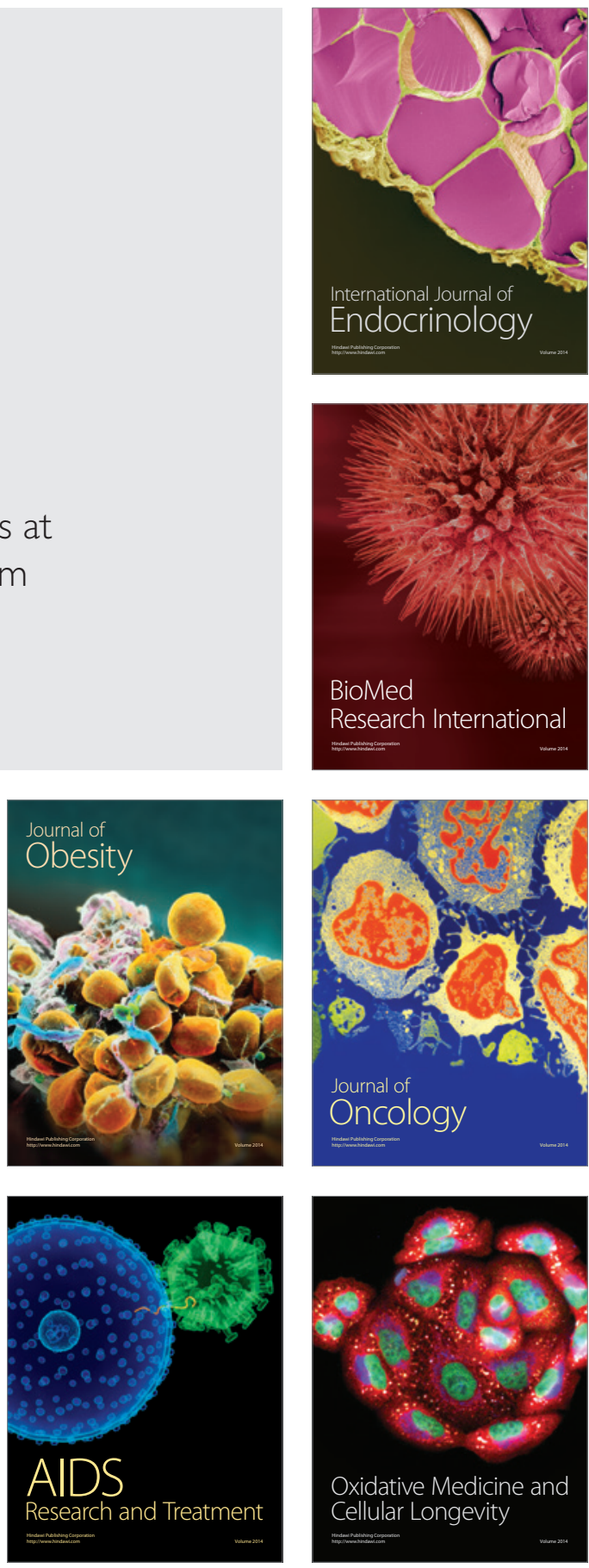